\title{
How to Perform and Assess Colonic Manometry and Barostat Study in Chronic Constipation
}

\author{
Yeong Yeh Lee, ${ }^{1,2 *}$ Askin Erdogan ${ }^{2}$ and Satish S C Rao ${ }^{2}$ \\ ${ }^{1}$ School of Medical Sciences, Universiti Sains Malaysia, KubangKerian, Kota Bahru, Kelantan, Malaysia; and ${ }^{2}$ Department of Medicine, Section \\ of Gastroenterology and Hepatology, Georgia Regents University, Augusta, Georgia, USA
}

\begin{abstract}
Management of chronic constipation with refractory symptoms can be challenging. Although new drugs and behavioral treatments have improved outcome, when they fail, there is little guidance on what to do next. At this juncture, typically most doctors may refer for surgical intervention although total colectomy is associated with morbidity including complications such as recurrent bacterial overgrowth. Recently, colonic manometry with sensory/tone/compliance assessment with a barostat study has been shown to be useful. Technical challenges aside, adequate preparation, and appropriate equipment and knowledge of colonic physiology are keys for a successful procedure. The test itself appears to be safe with little complications. Currently, colonic manometry is usually performed with a 6-8 solid state or water-perfused sensor probe, although high-resolution fiber-optic colonic manometry with better spatiotemporal resolutions may become available in the near future. For a test that has evolved over 3 decades, normal physiology and abnormal findings for common phenotypes of chronic constipation, especially slow transit constipation, have been well characterized only recently largely through the advent of prolonged 24-hour ambulatory colonic manometry studies. Even though the test has been largely restricted to specialized laboratories at the moment, emerging new technologies and indications may facilitate its wider use in the near future.
\end{abstract}

(J Neurogastroenterol Motil 2014;20:547-552)

Key Words

Barostat; Colon; Constipation; Manometry

\section{Introduction}

Chronic constipation is a common problem in adults and children encountered by primary care physicians and gastroenterologists, affects $15 \%$ of population in Western and Asian countries and while most respond to effective therapies, there are approximately $2-5 \%$ patients with intractable symptoms requir- ing further evaluation. ${ }^{1}$ Transit studies, while useful, do not allow one to determine the underlying pathophysiology of refractory constipation. Clearly, at this stage one needs to know if the colonic dysfunction is severe enough warranting a colectomy or if the colonic function is well-preserved such that the constipation may respond to aggressive drug and behavioral measures. Under these circumstances, an assessment of colonic manometry with barostat evaluation may play an important role. ${ }^{2}$ Besides determining the

Received: April 30, 2014 Revised: May 9, 2014 Accepted: May 12, 2014

(c) This is an Open Access article distributed under the terms of the Creative Commons Attribution Non-Commercial License (http://creativecommons. org/licenses/by-nc/3.0) which permits unrestricted non-commercial use, distribution, and reproduction in any medium, provided the original work is properly cited.

*Correspondence: Yeong Yeh Lee, MD, PhD, FACP, FRCP, FACG

School of Medical Sciences, Universiti Sains Malaysia, 16150 KubangKerian, Kelantan, Malaysia

Tel: +609-767-6590, Fax: +609-767-3949, E-mail: justnleeyy@gmail.com

Financial support: None.

Conflicts of interest: None.

Author contributions: Yeong Yeh Lee, Askin Erdogan and Satich S C Rao contributed equally to the design, analysis and writing of the manuscript. ORCID: Yeong Yeh Lee, http://orcid.org/0000-0002-6486-7717. 
underlying pathophysiology, colonic manometry can provide subsequent guidance for further management that may include biofeedback or surgery.

\section{Test Equipment}

The manometer is commonly available in two kinds; one, a solid-state probe with multiple strain gauge microtransducers and two, a water-perfused probe that is usually used in the pediatric setting. The catheter is then connected to a pressure recording device and a computer for displaying measurement and for data storage.The solid-state probe has the advantage for studying patients over a longer period of time and in more physiological ambulatory conditions and frees up time and space in a motility lab. The downside is the higher initial costs and the probe is more fragile. Water-perfused probe requires a pneumohydraulic pump to continuously perfuse water through the side holes of the catheter and some concerns have been raised about excess water infusion. Hence, patients are confined to bed in a laboratory; since they are not able to ambulate, prolonged study is not possible. There are no studies that directly compare between the 2 techniques but the ability to perform ambulatory studies with solid-state probe is clearly advantageous.

In adults, a probe with 6 to 8 sensors, with sensor spacing between 10 to $20 \mathrm{~cm}$ is useful so that the propagating pressure waves can be recorded from the ascending colon, transverse colon, descending colon, sigmoid colon and the rectum (Fig. 1). In pediatrics, the distance between recording sites using a probe with 6 to 8 sensors is typically 5 to $15 \mathrm{~cm}$. A recent study suggests that majority of propagating events propagate $<10 \mathrm{~cm}$ along the colon questioning the accuracy of measurements using "low-resolution" catheters with widely spaced sensors. ${ }^{3}$ Newer "high-resolution" catheter is now available using fiber-optic technology allowing 72 to 90 sensors (up to 144 sensors is possible) on a probe with each sensor spaced at $1 \mathrm{~cm}$ intervals. ${ }^{3}$ Singh et al ${ }^{2}$ reported that $41 \%$ of patients with slow transit constipation (STC) had normal colonic motility when using catheter with 6 sensors spaced between 10 and $20 \mathrm{~cm}$. This suggests that "low-resolution" colonic manometry may not be able to differentiate controls from certain patients with known motility disorders. Recently, Dinning et $\mathrm{al}^{4}$ reported that closely spaced sensors can avoid gross misinterpretation of frequency, morphology and directionality of colonic propagating sequences. However, how the new technology translates into clinical practice remains to be defined. Furthermore, the catheter is not commercially available. Also, the analysis can be cumbersome with a large volume of physiological data. More clinical studies regarding its advantage, clinical significance and robustness are awaited.

To assess for colonic tone and sensation, a barostat assembly can be incorporated with the manometer. ${ }^{5}$ The assembly consists of a $10 \mathrm{~cm}$ long highly compliant balloon, and this balloon is placed under colonoscopic or fluoroscopic guidance so that it sits at the mid-descending colon.

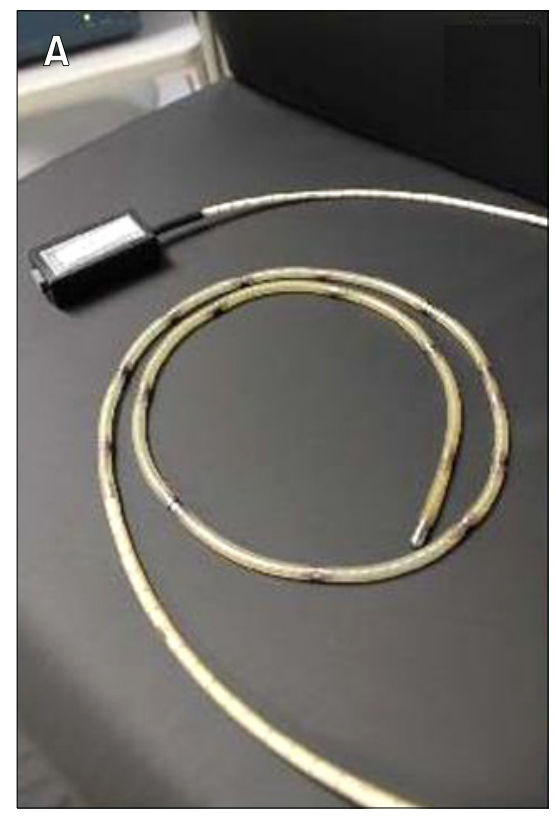

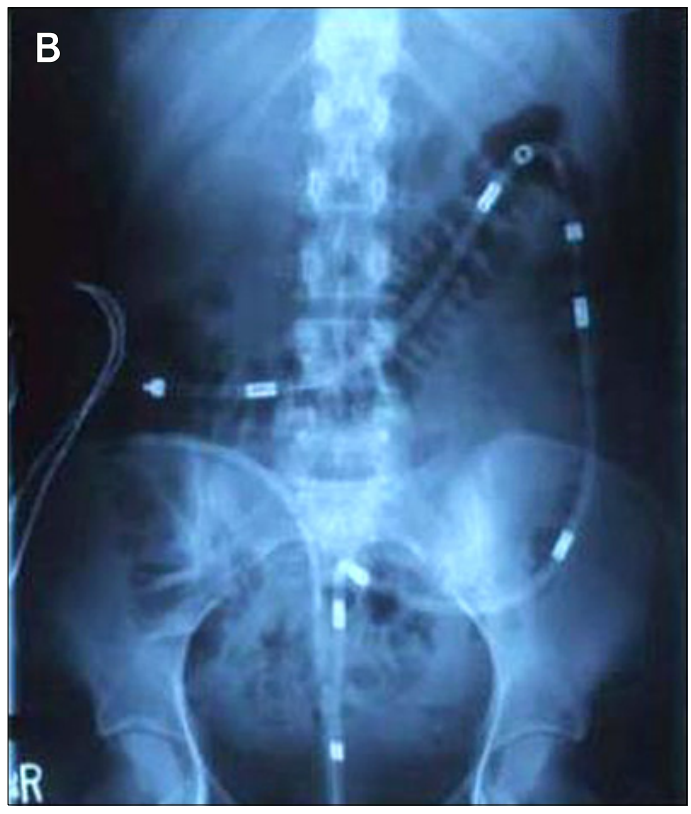

Figure 1. Colonic manometry assembly (A) and abdominal X-ray (B) showing typical location of sensors after placement. 


\section{Test Procedure}

\section{Preparation and Placement}

Patients are advised to stop medications that may affect gastrointestinal motility for at least 48 hours before the test. These medications may include opioids (e.g., codeine and morphine), sedatives or tranquilizers (e.g., diazepam and amitriptyline), antispasmodics (e.g., dicyclomine and hyoscine) and prokinetics (e.g., erythromycin, domperidone and prucalopride) and laxatives and/or secretagogues (e.g., lubiprostone and linaclotide). Standard bowel preparation that includes polyethylene glycol and/or tap water enemas may be used but this may potentially increase frequency of contractions. ${ }^{6}$ After an overnight fast, the patient will arrive at the endoscopy suite in the morning. The procedure typically only requires minimal sedation. Propofol sedation may be preferable because it does not affect motility.

Although the probe can be placed either anally or orally, the anal route is preferable. Occasionally, the probe can be placed through a stoma. The anal route is commonly achieved through the use of a colonoscope under direct vision with the tip of probe positioned at or beyond the hepatic flexure. Hemostatic clips have been successfully used to anchor the manometer over silk sutures located at the tip and 3 other sites. ${ }^{7}$ After colonoscopic placement, position of the catheter should be verified either with fluoroscopy or with abdominal X-ray. For ambulatory studies, the solid state probe should be connected to a recording device, which is then placed in a small carrying bag.

\section{Study Protocol}

A rest period of 2 to 5 hours is usually required prior to starting actual physiological recording. This will reduce the effects of bowel preparation, endoscopy, and administration of sedating drugs. Standard meals are allowed during the 24-hour ambulatory period, as well as other normal activities including sleeping. Patients are encouraged to wake up at standard times, and strictly record their symptoms/activities on a stool/symptom diary and with the use of event buttons provided on the recorder.

Provocation studies are short duration static recordings to assess the effect of stimulus on colonic motor activity. These stimuli may be pharmacologic, for example, bisacodyl, cholinergic agents (neostigmine) and/or a standardized meal (for example, roast beef sandwich with mayonnaise, milkshake, vanilla ice-cream and a non-caloric beverage, with a total caloric value of $1,060 \mathrm{kcal})$.
Testing for colonic sensation with the barostat is usually performed the next day, when the patient returns to the lab. After completing the study, the probe can be removed slowly and carefully by tugging it out.

\section{Data Analysis}

All symptoms and ambulatory activities recorded in the patient's diary should be entered into the program prior to starting analysis. The pressure activities should be analyzed in detail for each sensor and in each hour, with attention to the following parameters; patterns, types and number of contractile waves, their area under the curve, amplitude, duration, direction, velocity and length of propagation. ${ }^{8}$ Likewise, pressure activities during provocation studies should be assessed in detail including meal-induced gastrocolonic response 1 hour and 2 hours after a standardized meal, waking-up response and variations in response to pharmacologic stimuli on the motility index. Colonic motor activity is complex, variable, exhibits diurnal and spatio-temporal variation. Seven different patterns of activity, namely high amplitude propagating contractions, propagating pressure waves, retrograde pressure waves, simultaneous contractions, periodic rectal motor activity, periodic colonic motor activity and isolated contractions have been described. Colonic manometry findings in adults have been shown to be generally reproducible with little intra and inter-individual variation, ${ }^{9}$ although in children, the gastrocolonic response may be less consistent. ${ }^{10}$

\section{Trouble-shooting During the Test and Complications}

Besides adequate bowel preparation, it is equally important to ensure the equipment in good working condition. New batteries should be used each time to power the recording device. The storage disk should be checked to ensure previous data have been saved and cleared before storing new ones. Manometer probeshould be inspected for any malfunctions, calibrated and sutureapplied at appropriate intervals along its length prior to insertion.

Placement of probe with colonoscopy may carry a risk of bleeding or perforation but this has not been reported with this procedure. Removal of probe upon completion of study should be performed with care to prevent any potential mucosal tear or bleeding from the clips.

It is important to educate patients on the care of probe during ambulatory studies, and emergency contact numbers should be provided. Occasionally, the probe may be accidentally pulled out 
or stopped working, in which case, patient should alert the staff immediately. Patient should not bathe during the test, and no strenuous activities or exercises are allowed. It must be stressed to patients on the importance for them to have a detailed recording of their symptoms/activities in the provided diary.

Table 1. Indications for Colonic Manometry

Adults

Chronic slow transit constipation that is not responsive to medical therapy in the absence of an evacuation disorder

Chronic colonic pseudo-obstruction and megacolon or megarectum, with viscus diameter exceeding 10 and $15 \mathrm{~cm}$ respectively

Children

As above

Persistent symptoms after surgery for Hirschprung's disease to determine underlying pathophysiology

Evaluate function of a diverting colon before possible closure

Predict response to antegrade enemas via cecostomy

\section{Indications}

Most patients with chronic constipation or pseudo-obstruction and suspected colonic dysmotility would have been assessed first by transit studies including radio-opaque markers, scintigraphic studies or more recently wireless motility capsule. ${ }^{11}$ These studies, although useful, do not provide a pathophysiological explanation of symptoms, and hence will not be adequate to guide subsequent management. Indications for colonic manometry are given in Table 1, based on recommendation in 2008 from the American Neurogastroenterology and Motility Society. ${ }^{5}$

\section{Analysis and Interpretations}

\section{Normal Physiology}

The left colon transports formed stools in the right colon into

Table 2. Normal and Abnormal Findings in Colonic Manometry and Barostat Studies

\begin{tabular}{|c|c|c|}
\hline Findings & Normal & Abnormal \\
\hline \multicolumn{3}{|l|}{ Phasic contractions } \\
\hline $\begin{array}{l}\text { High amplitude propagating } \\
\text { contractions (HAPCs) }\end{array}$ & $\begin{array}{l}\text { Amplitude }>50-100 \mathrm{mmHg}, 4-10 / \text { day, mostly after meal } \\
\text { and awakening, propagate over } 3 \text { or more sites, duration } \\
\geq 14 \text { seconds, arise proximally, seldom migrate beyond } \\
\text { mid-colon, velocity } 1.5 \mathrm{~cm} / \mathrm{sec} \text { right and left side of colon, } \\
\text { antegrade or retrograde. }\end{array}$ & $\begin{array}{l}\text { Absent HAPCs or reduce in frequency, amplitude } \\
\text { and velocity in STC. HAPCs are more frequent } \\
\text { in IBS, especially sigmoid colon, reached more } \\
\text { distally, and in clusters. Less augmentation in } \\
\text { frequency and amplitude of HAPCs preceding } \\
\text { defecation in DD. }\end{array}$ \\
\hline $\begin{array}{l}\text { Low amplitude propagating } \\
\text { contractions (LAPCs) }\end{array}$ & $\begin{array}{l}\text { Amplitude }<50 \mathrm{mmHg} \text { with mean of } 20 \mathrm{mmHg}, 60 / \text { day, } \\
\text { more frequent after meal and upon awakening, actual } \\
\text { physiology not clear. }\end{array}$ & $\begin{array}{l}\text { Less frequency in STC but not IBS-C. Increase } \\
\text { in LAPCs after meals in both STC and IBS-C. }\end{array}$ \\
\hline $\begin{array}{l}\text { Periodic retrograde rectal } \\
\text { motor activity (PRMA) }\end{array}$ & $\begin{array}{l}\text { Retrograde, propagating or non-propagating, arise at } \\
\text { rectosigmoid colon, more common at night, lasts } \geq 3 \\
\text { minutes, frequency } 3 \text { waves } / \text { min. }\end{array}$ & $\begin{array}{l}\text { Reduced daytime activity but higher frequency } \\
\text { and are more uncoordinated in STC. }\end{array}$ \\
\hline \multicolumn{3}{|l|}{ Provocative studies } \\
\hline Gastrocolonic response to food & $\begin{array}{l}\text { Two fold increase in contractions in the first } 2 \text { hour vs. } 1 \\
\text { hour before food. }\end{array}$ & Absent or reduced $(<15 \%)$ in HAPCs in STC. \\
\hline Upon awakening & $\begin{array}{l}\text { Three fold increase in contractions in the first hour vs. } \\
\text { 1-hour pre-wake. }\end{array}$ & Absent or reduced in HAPCs in STC. \\
\hline $\begin{array}{l}\text { Endoluminal instillation of } \\
\text { bisacodyl }(10-20 \mathrm{mg}) \text { and } \\
\text { intravenous neostigmine }(1.5 \mathrm{mg})\end{array}$ & $\begin{array}{l}\text { Activate myenteric plexus to produce propagating contrac- } \\
\text { tions in normal individuals }\end{array}$ & No increase in HAPCs in STC after 30 minutes. \\
\hline Colonic tone and compliance & $\begin{array}{l}\text { Increase tone in response to food, more in transverse colon } \\
\text { than in sigmoid colon (mean } 24 \% \text { vs. } 13 \% \text { increase in } 90 \\
\text { min). }\end{array}$ & $\begin{array}{l}\text { Reduce tone in STC, DD and NTC. Reduce in } \\
\text { compliance in STC and DD but not NTC. }\end{array}$ \\
\hline Colonic sensation & $\begin{array}{l}\text { Normal threshold pressures to first sensation, desire, urge, } \\
\text { maximal tolerable pressure. }\end{array}$ & Hyposensitive in STC and hypersensitive in IBS. \\
\hline
\end{tabular}

STC, slow transit constipation; IBS, irritable bowel syndrome; DD, dyssynergic defecation; IBS-C, constipation predominant IBS; NTC, normal transit constipation. 
the rectosigmoid area, which is the main sensorimotor region that facilitates voluntary evacuation of stool. Colonic manometry measures the phasic contractions and barostat assembly, the tone and sensation (Table 2). The phasic contractions have been shown to be highly complex, intermittent and variable in healthy subjects, but it can be broadly divided into propagating or non-propagating/segmental. Propagating contractions can be either low or high amplitude ( $>50 \mathrm{mmHg}$ ), with high-amplitude propagating contractions (HAPCs), having a more functional and pathological significance. These contractions may be migrating either retrograde or antegrade in direction, and in contrary to previous reports, using high-resolution catheters, retrograde contractions appear to be more frequent than antegrade contractions. ${ }^{4}$ Presence of retrograde contractions especially in the distal colon plays a role in retarding flow. Periodic rectal motor activity (PRMA) is another type of motor activity seen in the rectosigmoid colon. PRMA is likely triggered by arrival of stool or gas in the rectum and may serve as a braking mechanism preventing untimely defecation. ${ }^{12}$

\section{Abnormal findings}

Abnormalities of phasic contractions, provocative studies, colonic tone, compliance and sensation in chronic constipation are shown in Table 2. The presence or absence of three cardinal responses namely, HAPCs, gastrocolonic response after standardized meal and waking-up response have been used to classify colonic manometry into normal, myopathic or neuropathic. With

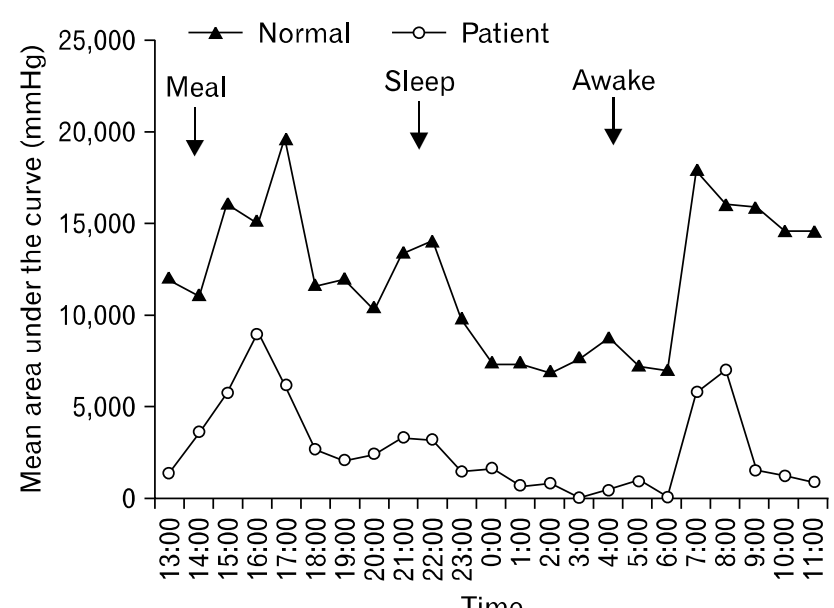

Figure 2. A 24-hour profile of mean area under the curve of colonic pressure waves in a healthy subject and in a constipated patient with colonic neuropathy. Note the marked impairment in meal-induced gastrocolonic response and waking response but a preserved diurnal variation in the patient.
STC, phasic motor activity is significantly impaired, especially HAPCs, where the frequency, amplitude and velocity tend to be dampened (Fig. 2). There is also absence or impairment of gastrocolonic response to meal and waking response. Examples of manometric patterns of normal, neuropathy and myopathy are shown in Figure 3. Colonic neuropathy is present if any 2 of the above 3 responses are absent. It is colonic myopathy if the magnitude of response for any 2 of the 3 responses is less than 2 standard deviations of the normal range. The presence of these distinct phenotypes has been reaffirmed in a recent study in adults ${ }^{2}$ and also in children. ${ }^{13}$ Cholinergic stimulation produces propagating contractions and/or HAPCs in normal colon but not in a neuropathic colon. The colonic tone is also reduced in chronic constipation, as well as reduced compliance in STC and dyssynergic defecation. ${ }^{14}$

\section{$A_{\text {Normal }}$}

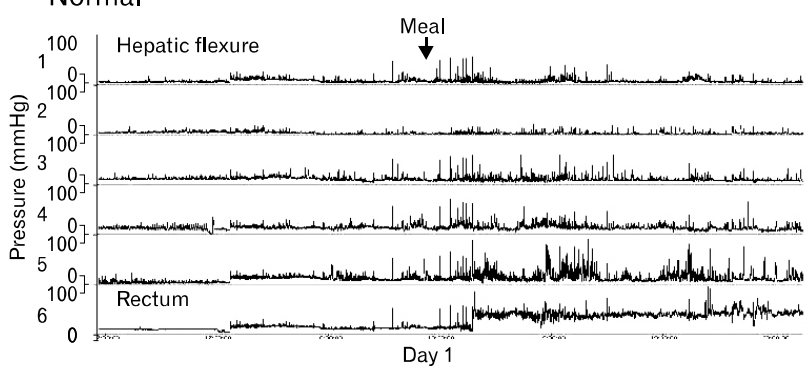

\section{B Myopathy}

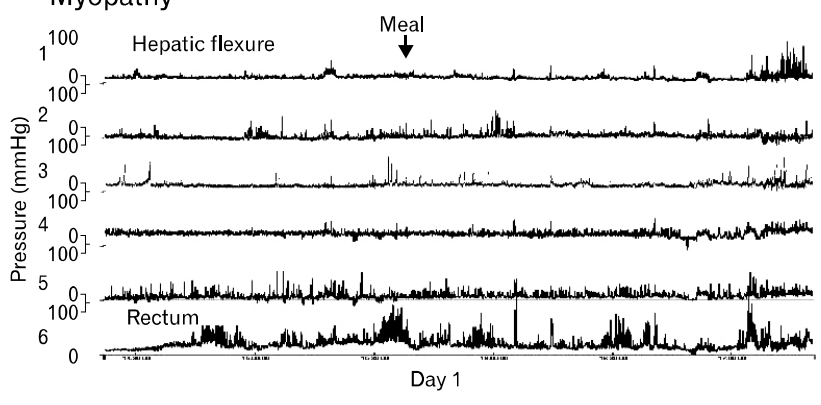

\section{Neuropathy}

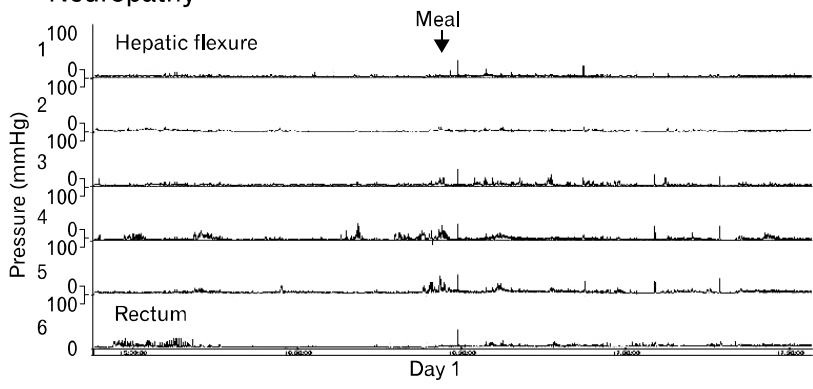

Figure 3. Manometric patterns of (A) normal, (B) colonic myopathy and $(\mathrm{C})$ colonic neuropathy in slow transit constipation. 
Patients with STC are more likely to be hyposensitive but those with irritable bowel syndrome would be more hypersensitive.

\section{How Clinically Useful Is Colonic Manometry in Practice?}

In STC, approximately a third of adult patients responded to pharmacological therapy alone, and half responded to pharmacological and biofeedback therapy. ${ }^{2}$ More patients with myopathy will respond to conservative measures including biofeedback than those with neuropathy. Of those patients with neuropathy and referred for surgical intervention, majority improve although half of these patients may develop postoperative complications, especially bacterial overgrowth. Provocative studies using bisacodyl or neostigmine may further assist in selecting patients with colonic inertia for surgery. ${ }^{5}$ Unfortunately, colonic manometry is only available in some specialized centers. In such situation, many doctors may decide to refer for colectomy when all therapeutic options have failed rather than referring them to these centers.

\section{Other Uses of Colonic Manometry}

Besides indications in Table 1 , in children with functional constipation, colonic manometry helps the child and family understand the maladaptive behaviors associated with functional symptoms. ${ }^{15}$ In some patients with functional diarrhea and associated autonomic neuropathy, more frequent HAPCs are found during the day and also postprandially. ${ }^{5}$ There are emerging new therapies in treating constipation including prokinetics, of which colonic manometry may have a role in understanding their mechanism of actions in clinical trials.

\section{Conclusions}

Colonic manometry and sensation/tone/compliance assessment with a barostat are useful tools for evaluating the underlying pathophysiology and causes of chronic constipation in patients with refractory symptoms, especially prior to performing a colectomy. With better spatiotemporal resolutions, high-resolution fiber-optic catheters may be more widely available in the near future. Adequate preparation is important prior to catheter placement and the test is relatively safe with little complications. Abnormal findings in phasic contractions, colonic tone, compliance and sensation have been well-characterized especially in
STC. Although largely restricted to specialized laboratories at the moment, emerging new technologies and indications may facilitate wider use of colonic manometry.

\section{References}

1. Lee YY. What's new in the toolbox for constipation and faecal incontinence? Front Med 2014;1:5.

2. Singh S, Heady S, Coss-Adame E, Rao SS. Clinical utility of colonic manometry in slow transit constipation. Neurogastroenterol Motil 2013;25:487-495.

3. Bampton PA, Dinning PG. High resolution colonic manometry--what have we learnt? - A review of the literature 2012. Curr Gastroenterol Rep 2013;15:328.

4. Dinning PG, Wiklendt L, Gibbins I, et al. Low-resolution colonic manometry leads to a gross misinterpretation of the frequency and polarity of propagating sequences: initial results from fiber-optic high-resolution manometry studies. Neurogastroenterol Motil 2013; 25:e640-e649.

5. Camilleri M, Bharucha AE, di Lorenzo C, et al. American Neurogastroenterology and Motility Society consensus statement on intraluminal measurement of gastrointestinal and colonic motility in clinical practice. Neurogastroenterol Motil 2008;20:1269-1282.

6. Dinning PG, Zarate N, Szczesniak MM, et al. Bowel preparation affects the amplitude and spatiotemporal organization of colonic propagating sequences. Neurogastroenterol Motil 2010;22:633, e176.

7. Rao SS, Singh S, Sadeghi P. Is endoscopic mucosal clipping useful for preventing colonic manometry probe displacement? J Clin Gastroenterol 2010;44:620-624.

8. Rao SS, Singh S. Clinical utility of colonic and anorectal manometry in chronic constipation. J Clin Gastroenterol 2010;44:597-609.

9. Rao SS, Singh S, Mudipalli R. Day-to-day reproducibility of prolonged ambulatory colonic manometry in healthy subjects. Neurogastroenterol Motil 2010;22:640, e178.

10. Sood MR, Mousa $\mathrm{H}$, Tipnis $\mathrm{N}$, et al. Interobserver variability in the interpretation of colon manometry studies in children. J Pediatr Gastroenterol Nutr 2012;55:548-551.

11. Lee YY, Erdogan A, Rao SS. How to assess regional and whole gut transit time with wireless motility capsule. J Neurogastroenterol Motil 2014;20:265-270.

12. Rao SS, Welcher K. Periodic rectal motor activity: the intrinsic colonic gatekeeper? Am J Gastroenterol 1996;91:890-897.

13. Giorgio V, Borrelli $\mathrm{O}$, Smith VV, et al. High-resolution colonic manometry accurately predicts colonic neuromuscular pathological phenotype in pediatric slow transit constipation. Neurogastroenterol Motil 2013;25:70-78, e8-e9.

14. Ravi K, Bharucha AE, Camilleri M, Rhoten D, Bakken T, Zinsmeister AR. Phenotypic variation of colonic motor functions in chronic constipation. Gastroenterology 2010;138:89-97.

15. Firestone Baum C, John A, Srinivasan K, et al. Colon manometry proves that perception of the urge to defecate is present in children with functional constipation who deny sensation. J Pediatr Gastroenterol Nutr 2013;56:19-22. 